\title{
Adsorption kinetics in the presence of external fields
}

\author{
I. Pagonabarraga, ${ }^{1, *}$ J. Bafaluy, ${ }^{2}$ and J. M. Rubi ${ }^{3}$ \\ ${ }^{1}$ FOM Institute for Atomic and Molecular Physics, Kruislaan 407, 1098 SJ Amsterdam, The Netherlands \\ ${ }^{2}$ Departament de Física, Universitat Autònoma de Barcelona 08193 Bellaterra, Barcelona, Spain \\ ${ }^{3}$ Departament de Física Fonamental, Universitat de Barcelona, Avinguda Diagonal 647, E-08028 Barcelona, Spain
}

(Received 19 October 1998)

\begin{abstract}
In this article we present a detailed analysis of the kinetics of a class of sequential adsorption models that take into account the effect of externally applied fields (as an electric field, or a shear rate) on the adsorption. The excluded volume interactions related to the finite size of the adsorbing particles are modified by the external fields. As a result, new adsorption mechanisms appear with respect to the ones used to describe the kinetics in a quiescent fluid. In particular, if the adsorbing particles are allowed to roll over preadsorbed ones, adsorption becomes non local even in the simplest geometry. An exact analytic theory cannot be developed, but we introduce a self-consistent theory that turns out to agree with the simulation results over all the range of the parameters. [S1063-651X(99)01904-2]

PACS number(s): 82.70.Dd, 68.45.Da
\end{abstract}

\section{INTRODUCTION}

The irreversible adsorption of colloidal particles on a solid surface is a complex process that depends both on interactions between the particles and on their actual motion through the solvent. From the different mechanisms that control the adsorption process, the excluded volume interactions related to the finite size of the particles [1] has attracted much attention. To this end, models have been introduced in which a detailed description of the actual adsorption mechanisms is disregarded, and the dynamics is described in terms of sequential kinetic models, according to which colloids are accepted or rejected at the surface on the basis of geometric rules. Although they constitute oversimplified models of the actual adsorption process, excluded volume effects are essential in the adsorption of colloids, and in fact agreement with experimental results is obtain for some quantities $[2,3]$. From the theoretical point of view, these models are interesting since analytic solutions can be found, and perturbative procedures are easily set up to deal with more general situations [4]. The standard model used to describe the adsorption of Brownian particles has been the random sequential adsorption model (RSA) [5], while the ballistic model (BM) has been proposed to describe adsorption controlled by gravity [6]. However, so far they have been compared with simple situations in which adsorption takes place in a quiescent fluid, in the absence of external forces, namely, diffusionand gravity-controlled adsorption. Recent experiments have been performed on the adsorption both of protein kinetic [7] and bacteria [8] structures when the solvent is subject to a shear flow, and there exist situations of practical interest where external fields are applied on the adsorbing suspension, as in capillary electrophoresis $[9,10]$, in which the adsorption of polarizable colloids on the walls of a capillary in the presence of electric fields is studied. In these cases, even

\footnotetext{
*Present address: Department of Physics and Astronomy, University of Edinburgh, JCMB King's Buildings, Mayfield Road EH93JZ, U.K.
}

without taking into account that the applied fields may induce the desorption of adsorbed particles, it is not clear that the geometric rules that characterize the standard sequential kinetic models can be applied to these new physical situations.

Although in fact the applied fields will modify the global transport of the particles from the bulk to the surface, the effect of more general transport conditions on the geometric exclusion effects has not been addressed systematically. In a previous paper [11], we have introduced a kinetic class of adsorption models that take into account the effect of an external field on the adsorption of colloidal suspensions. Due to the external field, the particles do not arrive following trajectories perpendicular to the surface. As a result, the area excluded by the preadsorbed colloids is asymmetric, and due to this effect we grouped this new family of models under the name of shadow models. Our main purpose in this paper is to study in detail such models, focusing on the modifications on the dynamical processes induced by the applied fields, and their effect on the kinetics of the shadow models.

We will focus on the $(1+1)$-dimensional version of different kinetic adsorption models in which particles do not arrive perpendicularly to the substrate [11]. Although it is a simplification, it will be possible to obtain exact theoretical expressions, and the insight gained by such an analysis can be used in the development of perturbative theories for more general cases [4]. In fact, most of the mechanisms we will discuss will be present for any dimensionality of the system.

Due to the presence of the external field, disks of unit diameter arrive at the line forming an angle $\alpha$ with the normal to the wall, as shown in Fig. 1(a). This angle $\alpha$ will be the only free parameter of the model. If a constant external field $F_{e}$ parallel to the surface is applied besides the usual gravitational force $F_{g}$, and neglecting hydrodynamic effects, then $\alpha$ is related to the physical parameters by $\tan \alpha$ $=F_{e} / F_{g}$. An equivalent relation is satisfied if the host fluid is subject to a plug flow. If instead, a Couette flow is applied in the absence of diffusion, then more care should be taken, because in this case the incoming particle will describe a parabola. Nonetheless, a relationship with the case of straight 


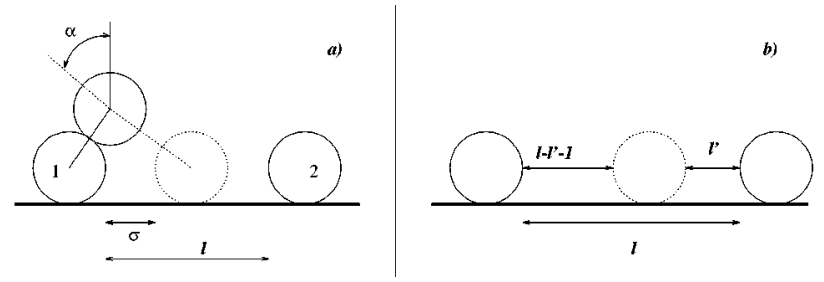

FIG. 1. (a) Trajectory of an incoming particle of diameter unity forming an angle $\alpha$ with the normal to the adsorbing line, in the presence of two preadsorbed disks, 1 and 2, at a distance $l$. The dotted line identifies the trajectory for which the incoming particle is tangent to the adsorbed one, and it is adsorbed at the minimum distance between the centers of $1+\sigma$. (b) New gaps appearing when an incoming particle lands at distance $l^{\prime}$ from the preadsorbed disk at right.

trajectories can be found if one takes into account that there is a minimum distance at which an incoming particle following a parabola can land in the nearby of a preadsorbed disk. Finally, if adsorption takes place in a quiescent fluid, in the presence of a gravity field, on a substrate that is inclined at an angle $\gamma$ with respect to the horizontal, then, one has obviously $\alpha=\gamma$. The three different physical situations are described by a single parameter $\alpha$, which gives an idea of the distance from the corresponding classical kinetic models.

Although the existence of an external field acting parallel to the substrate or the shearing of the solvent correspond to different physical situations, and the detailed transport mechanisms will differ, from the point of view of the volume excluded by preadsorbed particles in both cases additional fractions of the substrate at one side of the preadsorbed particles become blocked for adsorption, and due to this asymmetry, it is not possible to reduce these situations to the standard RSA or BM by rescaling of the lengths of the particles. We will separately analyze both the case where incoming particles are not allowed to roll over preadsorbed ones, corresponding to RSA type rules, and the one where rolling is permitted, which is then equivalent to BM. In the next section we will study the generalized RSA model, considering both the behavior of global properties and the local distribution of particles, while in the third section we will focus on the ballistic model, where new adsorption processes take place. Already for this model an exact solution does not always exist. We present a self-consistent theory and compare with simulation results. Finally, in the conclusions we present our main results.

\section{INCLINED RANDOM SEQUENTIAL ADSORPTION MODEL}

We will first concentrate on the model where adsorption kinetics proceeds according to RSA rules. In the standard RSA model, one usually starts with an empty line, and positions for the centers of the disks are chosen uniformly at random along the substrate. The particle sticks irreversibly at the chosen position if no overlap exists with a preadsorbed disk, where overlap means that the distance from the center of the incoming particle to any of the preadsorbed ones is smaller than the diameter of the disks; otherwise the trial position is rejected and a new position is chosen uniformly at random along the line. The process is repeated until no more particles can be placed on the substrate; this final state is referred to as the jamming.

We now consider that disks of unit diameter arrive at the substrate at rate $k$ per unit length along trajectories that form an angle $\alpha$ with the normal to the wall, as shown in Fig. 1. For simplicity, the rate will be taken $k=1$ in the rest of the paper. Due to the inclination of the arrival trajectories, a new minimum distance between disks on the surface, $\sigma$, appears. As can be seen in Fig. 1, an incoming particle will now overlap a preadsorbed one if its trajectory ends on the substrate to the right of the preadsorbed particle at a center-tocenter distance smaller than $1+\sigma$. As can be deduced from the geometry of Fig. 1, $\sigma$ is related to the angle of inclination of the trajectory through $1+\sigma=1 / \cos \alpha$. This additional excluded distance can be looked as a shadow cast by the preadsorbed particle.

The kinetic rules are the same as in standard RSA, after appropriately modifying the overlap mechanism. Now, a trial position is accepted if the distance to the center of the nearest preadsorbed disk on its right-hand side is larger than unity, and the distance to the nearest pre-adsorbed particle on its left-hand side is larger than $1+\sigma$. It is worth noting that this description of the model in terms of two excluded lengths is equivalent to a restructuring model in which incoming particles of length one, upon arrival at the surface, deform a length $\sigma$ towards the right. This asymmetry in the deformation makes these new models different from the restructuring-particle RSA proposed recently to describe the adsorption of certain proteins that undergo structural changes once on the substrate [12]. Also, a recent model for the adsorption of "hot" particles takes into account the motion of particles after being adsorbed [13]. However, the rules differ from the ones we consider here.

For the 1+1-dimensional model it is possible to obtain an analytic expression for the evolution of the one-gap density function, $G(l, t)$, i.e., the probability density of having gaps of length $l$ at time $t$. This is due to the shielding property, according to which the evolution of one gap is independent of the others, since the particle separating two gaps prevents any interaction between them. This property is lost at higher dimensions. We will follow the method introduced to study $\mathrm{BM}$ [6], according to which the evolution equations for the probability densities can be written once the gap creation and destruction mechanisms have been identified. Therefore, we will first determine the different ways in which a gap of a given length $l$ can be created and destroyed, and at which rate.

Let us consider a gap of length $l$. Any incoming particle should be at a minimum separation $\sigma$ at the right of the particle which delimits the gap at its left-hand side. Then, if $l<1+\sigma$, any incoming disk in this gap will overlap at least with one of the disks that delimit it, and will therefore be rejected. This implies that gaps of length smaller than 1 $+\sigma$ cannot be destroyed by adsorption of an incoming particle. Since initial positions are chosen uniformly along the substrate, a gap of length $l>1+\sigma$ will be destroyed at a rate proportional to $l-\sigma-1$, which is the length of the available part of the gap where the center of an incoming particle can arrive without overlapping with any of the disks that delimit it. When a gap of length $l$ is destroyed, two new gaps are created, one of length $l^{\prime}$ to the right of the incoming particle, 
which can have any value smaller than $l-\sigma-1$, and another one of length $l-l^{\prime}-1$ at its left, which can have a minimum value of $\sigma$ and a maximum of $l-1$, as can be seen in Fig. 1(b). Note that the new gaps may be either larger or smaller than $1+\sigma$. Accordingly, the appropriate evolution equations for the one-gap density $G(l, t)$ read

$$
\begin{gathered}
\frac{\partial G(l, t)}{\partial t}=-(l-\sigma-1) G(l, t)+\int_{l+1}^{\infty} G\left(l^{\prime}, t\right) d l^{\prime} \\
+\int_{l+\sigma+1}^{\infty} G\left(l^{\prime}, t\right) d l^{\prime}, \quad l \geqslant 1+\sigma ; \\
\frac{\partial G(l, t)}{\partial t}=\int_{l+1}^{\infty} G\left(l^{\prime}, t\right) d l^{\prime}+\int_{l+\sigma+1}^{\infty} G\left(l^{\prime}, t\right) d l^{\prime}, \\
\frac{\partial G(l, t)}{\partial t}=\int_{l+\sigma+1}^{\infty} G\left(l^{\prime}, t\right) d l^{\prime}, \quad l \leqslant l \leqslant \sigma+1 ;
\end{gathered}
$$

The destruction term in Eq. (2.1) takes into account that gaps with $l>1+\sigma$ are destroyed at a rate proportional to the number of such gaps and to the length on which an incoming disk can adsorb, $l-\sigma-1$. On the other hand, gaps of length $l$ may be created from gaps of length $l^{\prime}$ at a rate proportional to the number of gaps of length $l^{\prime}$, taking into account that if the new gap is created on the left-hand side of the incoming particle, the length of the initial gap should be at least $l$ +1 , while if it is created on the right-hand side of the incoming disk, the minimum necessary length is $l+\sigma+1$. However, when a particle arrives at the substrate, it must be at a minimum distance $\sigma$ from its left neighbor, and therefore gaps smaller than $\sigma$ will only be created on the right-hand side of incoming disks. For this the reason, Eq. (3) contains only the term corresponding to the creation of gaps to the right of the new particles.

Equations (2.1)-(2.3) constitute a set of integrodifferential equations that completely determine $G(l, t)$, once the appropriate initial conditions are prescribed. To avoid confusion in the notation, we will write down $G_{1}(l, t)$ when we refer to the expression of the gap density for $l \geqslant 1$ $+\sigma, G_{2}(l, t)$ for $\sigma \leqslant l \leqslant 1+\sigma$, and $G_{3}(l, t)$ for $l \leqslant \sigma$. As usual, we will consider that initially the line is empty, which implies $G(l, 0)=0$. Since the total length of the substrate is constant, the gap density must satisfy the normalization

$$
\int_{0}^{\infty}(l+1) G(l, t) d l=1 \text {. }
$$

The kinetics of the model is completely described in terms of the one-gap distribution function. For example, using the fact that the adsorption rate is proportional to the available fraction of surface, $\phi(t)$, the adsorption rate can be expressed as a quadrature,

$$
\phi(t) \equiv \frac{d \theta}{d t}=\int_{1+\sigma}^{\infty}(l-1-\sigma) G_{1}(l, t) d l .
$$

To solve Eqs. (2.1)-(2.4) we try the same "ansatz', for the gap density already proposed when solving the RSA model [6],

$$
G_{1}(l, t)=t^{2} e^{-(l-\sigma-1) t} F(t),
$$

where the factor $t^{2}$ has been introduced for convenience. Substituting this ansatz in Eq. (2.1) gives an ordinary differential equation for $F(t)$ that can be solved imposing the initial condition $F(0)=1$ consistent with an initially empty substrate, yielding

$$
\begin{aligned}
F(t) & =\exp \left\{-\int_{0}^{t} \frac{1-e^{-u}}{u} d u-\int_{0}^{t} \frac{e^{-(1+\sigma) u}}{u} d u\right\} \\
& =\frac{e^{-2 \gamma}}{(1+\sigma) t^{2}} e^{-E_{1}(t)-E_{1}[(1+\sigma) t]}
\end{aligned}
$$

where $E_{1}(t)$ is the exponential-integral function [14]. Once $G_{1}(l, t)$ has been determined, Eqs. (2.2) and (2.3) become differential equations for $G_{2}(l, t)$ and $G_{3}(l, t)$, which can be expressed as quadratures,

$$
\begin{gathered}
G_{2}(l, t)=\int_{0}^{t} \tau F(\tau) e^{-l \tau}\left\{1+e^{\sigma \tau}\right\} d \tau, \\
G_{3}(l, t)=\int_{0}^{t} \tau F(\tau) e^{-l \tau} d \tau .
\end{gathered}
$$

It is worth noting that $G_{2}(l, t)$ can be written in terms of $G_{3}(l, t)$, through the relation

$$
G_{2}(l, t)=G_{3}(l, t)+G_{3}(l-\sigma, t) .
$$

For arbitrary $l$, the function $G_{3}(l, t)$ gives the density of gaps of length $l$ created until time $t$ on the right-hand side of incoming disks, while $G_{2}(l, t)$ gives the total density of gaps of length $l$, irrespective of the side on which they have been created. Therefore, Eq. (2.10) shows that the number of gaps of length $l>\sigma$ created to the left of incoming particles is equal to the number of gaps of length $l-\sigma$ created to the right.

Equations (2.6), (2.8), and (2.9) completely define the one-gap number density at any time. At infinite time, when the jamming state is reached and no more disks can be adsorbed on the substrate, the gap density is given by

$$
\begin{gathered}
G_{1}^{\infty}(l)=0, \quad l \geqslant 1+\sigma ; \\
G_{2}^{\infty}(l)=G_{3}^{\infty}(l)+G_{3}^{\infty}(l-\sigma), \quad 1+\sigma \geqslant l \geqslant \sigma ; \\
G_{3}^{\infty}(l)=\frac{e^{-2 \gamma}}{1+\sigma} \int_{0}^{\infty} d t \frac{e^{-l t}}{t} e^{-E_{1}(t)-E_{1}[(1+\sigma) t]}, \quad l \leqslant \sigma .
\end{gathered}
$$

which shows that only the gaps with $l<1+\sigma$, which cannot be destroyed, survive at jamming. As happens in the RSA 
model, $G^{\infty}(l)$ diverges logarithmically when $l \rightarrow 0^{+}$, as can be seen rewriting Eq. (2.13) as

$$
\begin{aligned}
G_{3}^{\infty}(l)= & \frac{1}{2(1+\sigma)}\left\{G_{\mathrm{RSA}}^{\infty}(l)+2 e^{-2 \gamma} \int_{0}^{\infty} \frac{e^{-l t}}{t} e^{-2 E_{1}(t)}\right. \\
& \left.\times\left(e^{E_{1}(t)-E_{1}[(1+\sigma) t]}-1\right) d t\right\}
\end{aligned}
$$

where $G_{\mathrm{RSA}}^{\infty}(l)$ is the gap density corresponding to standard RSA [5]. To lowest order in $l$ one has now

$$
\begin{aligned}
G_{3}^{\infty}(l)= & \frac{e^{-2 \gamma}}{1+\sigma}\{-\ln (l)-\gamma \\
& +\int_{0}^{1} \frac{1}{t} \exp \left[-E_{1}((1+\sigma) t)-E_{1}(t)\right] d t \\
& \left.+\int_{1}^{\infty} \frac{e^{-E_{1}((1+\sigma) t)-E_{1}(t)}-1}{t} d t\right\}+O(l),
\end{aligned}
$$

displaying the same logarithmic divergence as in standard RSA [15], although its amplitude is modulated by a factor $1 / 2(1+\sigma)$ with respect to the RSA value. Note that even in the limit $\sigma \rightarrow 0$ this amplitude is half the RSA value due to the fact that gaps of length smaller than $\sigma$ can only be created at the right of an incoming disk, instead of being created at both sides. On the other hand, the function $G_{2}^{\infty}(l)$ exhibits a similar logarithmic divergence for $l=\sigma^{+}$, as can be seen from Eq. (2.10). This means that, unlike standard RSA, two logarithmic divergences appear in $G^{\infty}(l)$ related to the fact that now there are two minimum distances at which disks can approach. Moreover, the additivity property Eq. (2.10) ensures that the divergences associated with the two minimum distances 0 and $\sigma$ exhibit the same divergent behavior, the divergence appearing at $l=\sigma^{+}$will being larger by the finite amount $G_{3}^{\infty}(\sigma)$ than the one at $l=0^{+}$. In the limit $\sigma$ $\rightarrow 0$ both peaks coalesce into a single one, which then becomes the one obtained in standard RSA.

Once we know the one-gap density function, using Eq. (2.5), we can express the adsorption rate as

$$
\frac{d \theta}{d t}=F(t)
$$

where we have made use of Eq. (2.6). This expression allows us to identify the function $F(t)$, introduced so far as a way to find the gap density, with the available line fraction, as happens in standard RSA. Equation (2.16) enables one also to express the coverage as a function of time as a quadrature, from which, in particular, the jamming coverage can be calculated, giving [11]

$$
\theta^{\infty}(\sigma)=\frac{e^{-2 \gamma}}{1+\sigma} \int_{0}^{\infty} \frac{e^{-2 E_{1}(t)}}{t^{2}} e^{-E_{1}((1+\sigma) t)+E_{1}(t)} d t
$$

If $\sigma \ll 1$, we may expand the second factor in the integral in powers of $\sigma$, leading to

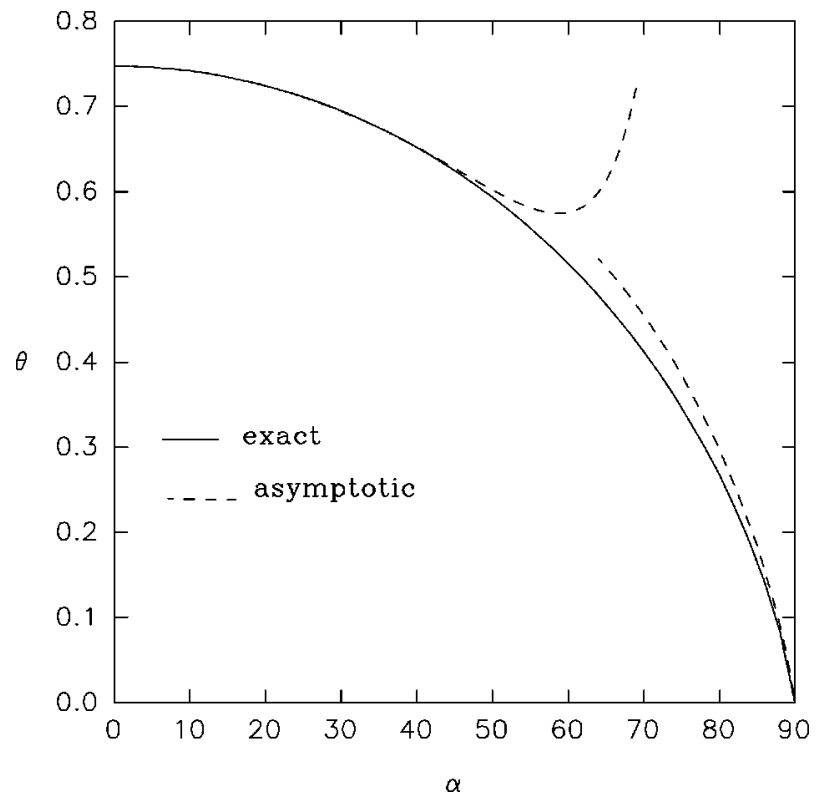

FIG. 2. The maximum coverage of the line as a function of the incident angle expressed in degrees. The dashed lines correspond to the expansion for small values of $\sigma$, up to the third order, and to the asymptotic expansion around $\alpha=90^{\circ}$ (see text).

$$
\begin{aligned}
\theta^{\infty}= & \theta_{\mathrm{RSA}}^{\infty}-\frac{\theta_{\mathrm{RSA}}^{\infty}}{2} \sigma+\left(\frac{3}{2} \theta_{\mathrm{RSA}}^{\infty}-e^{-2 \gamma}\right) \frac{\sigma^{2}}{4} \\
& -\left(\theta_{\mathrm{RSA}}^{\infty}-\frac{7}{3} e^{-2 \gamma}\right) \frac{\sigma^{3}}{4}+O\left(\sigma^{4}\right) \\
= & 0.747598-0.373799 \sigma+0.201540 \sigma^{2}+0.003011 \sigma^{3} \\
& +O\left(\sigma^{4}\right),
\end{aligned}
$$

where $\theta_{\mathrm{RSA}}^{\infty}$ is the jamming coverage for standard RSA, and the numerical values are numerical evaluations of the exact expression to give an idea of the magnitude of the corrections in the expansion. In Fig. 2 one can see that this expansion up to third order in powers of $\sigma$ agrees reasonably well with the exact results up to angles of the order of $40^{\circ}$.

We can also analyze the behavior of the jamming coverage when the adsorbing particles follow trajectories almost parallel to the substrate. In this case, one should take into account that the integral in Eq. (2.17) has a logarithmic singularity in the limit $\sigma \rightarrow \infty$, and a simple power expansion of the integrand cannot be performed. Instead, a matching asymptotic expansion method should be developed, in order to capture the different behavior of the integrand for small and large values of time [16]. One obtains that the jamming coverage vanishes as

$$
\theta^{\infty}(\sigma)=e^{-\gamma} \frac{\ln (1+\sigma)}{1+\sigma}+\frac{\omega}{1+\sigma}+O\left(\frac{1}{(1+\sigma)^{2}}\right)
$$

where the coefficient $\omega$ is 


$$
\begin{aligned}
\omega= & e^{-\gamma} \int_{0}^{1} \frac{e^{-E_{1}(t)}}{t} d t-e^{-\gamma} \int_{1}^{\infty} \frac{1-e^{-E_{1}(t)}}{t} d t \\
& +e^{-2 \gamma} \int_{1}^{\infty} \frac{e^{-E_{1}(t)}}{t^{2}} d t-e^{-\gamma} \int_{0}^{1} \frac{d t}{t}\left[1-\frac{e^{-\gamma}}{t} e^{-E_{1}(t)}\right] \\
= & 0.725533 .
\end{aligned}
$$

Again, in Fig. 2, it is shown that the asymptotic prediction works well for angles close to $90^{\circ}$. Although it is not possible to express the integral of Eq. (2.16) as a combination of known functions, it is of great help when trying to understand the filling kinetics. For example, we can analyze both the short time and the asymptotic behavior of $\theta(t)$. For short times the integral can be developed in powers of time,

$$
\theta(t)=t-\left(1+\frac{\sigma}{2}\right) t^{2}+\left(5+5 \sigma+\frac{3}{2} \sigma^{2}\right) \frac{t^{3}}{6}+O\left(t^{4}\right),
$$

showing that, initially, $\theta$ evolves as a power law, and differences with respect to standard RSA appear in the second power of time, because one should wait at least to the deposition of the second particle on the line to detect the effects of the new restrictions on the deposition of particles. For large values of $t$, the jamming coverage is asymptotically approached in the form

$$
\theta_{\infty}-\theta(t)=\frac{e^{-2 \gamma}}{(1+\sigma) t}\left(1-\frac{e^{-t}}{t}\right)+O\left(\frac{e^{-t}}{t^{3}}\right)
$$

which exhibits a power-law decay to the leading order. This reflects the fact that the size of the available areas for incoming particles approach zero asymptotically in the neighborhood of the jamming limit. This behavior is related to the logarithmic divergence of the gap density, and contains the same prefactor $1 /(1+\sigma)$ with respect to the RSA case.

As a final point regarding global quantities, it is possible to calculate perturbatively the available line fraction as a function of the coverage, which is of interest since it is more directly related to the properties of the filling process [17]. One gets

$\phi(\theta)=1-(2+\sigma) \theta+\left(1+\sigma+\frac{\sigma^{2}}{2}\right) \frac{\theta^{2}}{2}+O\left(\theta^{3}\right)$.

At zero coverage this quantity is equal to 1 since initially the line is empty, and any incoming particle is adsorbed. The second term simply shows that one adsorbed disk excludes an area equal to twice its diameter plus the additional exclusion length $\sigma$. The next term takes into account that the total length excluded by two adsorbed disks becomes more complex since their exclusion regions can overlap. In fact, the available fraction of the line up to order $\theta^{n}$ can be constructed by looking at the different ways in which $n$ disks can overlap [18,4]. Widom [17] showed that the functions $\phi$ for RSA and equilibrium coincide up to the second order in $\theta$. We can also compare Eq. (2.23) with the available line fraction for an equilibrium system of hard particles. Since in our model one particle excludes a length $2+\sigma$, we choose a system of particles of length 1 but interacting with a hard- core potential of range $1+\sigma / 2$. The equilibrium result is the same function of the coverage than for simple hard rods, but multiplying the coverage by a factor $(1+\sigma / 2)$. One then obtains

$\phi_{e q}(\theta)=1-(2+\sigma) \theta+\left(1+\sigma+\frac{\sigma^{2}}{4}\right) \frac{\theta^{2}}{2}+O\left(\theta^{3}\right)$

which shows that the available line function in the inclined RSA model deviates from the equilibrium one already in the second power in $\theta$, while in standard RSA differences appear in the third power of $\theta$. This is due to the asymmetry introduced in the kinetics by the inclined direction of arrival. In standard RSA, the configurations generated by two particles are equivalent to the equilibrium ones, in the sense that one cannot discern which of the two particles has been adsorbed first. In inclined RSA, however, not all the configurations with two adsorbed particles have the same probability than in equilibrium: if the separation between them is smaller than $\sigma$, then the particle at the right must have arrived before than the particle at the left, and this configuration has a probability that is one-half of the equilibrium value. This asymmetry in the kinetics has no analogy in equilibrium, and the behavior of the system deviates from equilibrium already at second order in $\theta$.

\section{INCLINED BALLISTIC DEPOSITION MODEL}

We will now consider a second kinetic adsorption model which evolves according to BM rules [6]. Since BM is intended to describe the adsorption of heavy colloids in the presence of gravity fields [6], this model can describe the adsorption in such a system when an additional force parallel to the surface, as for example an electric one, acts on the suspended particles. We will analyze in detail the new adsorption mechanisms induced by the presence of this new field. In order to describe them properly, we will first focus on the possible trajectories after one or a couple of particles have been adsorbed.

As in RSA the adsorption is still sequential, but when an incoming disk touches a preadsorbed one it is not rejected. Rather, it rolls over the latter trying to reach the substrate. Only if it gets trapped in the space between two adsorbed particles, being unable to reach the line, it is rejected. Since the trajectories are inclined with respect to the substrate, an incoming disk rolling over a preadsorbed one will move to its left-hand side if its initial trajectory ends to the left of the center of the preadsorbed particle, and it will roll to the right otherwise. Both kinds of trajectories can be delimited by a separation line $\Gamma$, starting at the center of the preadsorbed particle and with the same inclination as the trajectories of the incoming disks. Note that in standard BM half of the incoming particles that overlap an adsorbed disk will try to reach the substrate on its right-hand side and half on its lefthand side. Now, due to the inclination of the separation line, a fraction $1 /(2+\sigma)$ of the incoming particles that overlap a preadsorbed disk will roll towards its left-hand side, while a fraction $(1+\sigma) /(2+\sigma)$ will roll towards its right.

If the center of the incoming disk is on the right-hand side of $\Gamma$ when touching disk 1 , it will roll towards the right-hand side of that particle, until its trajectory becomes tangent to 


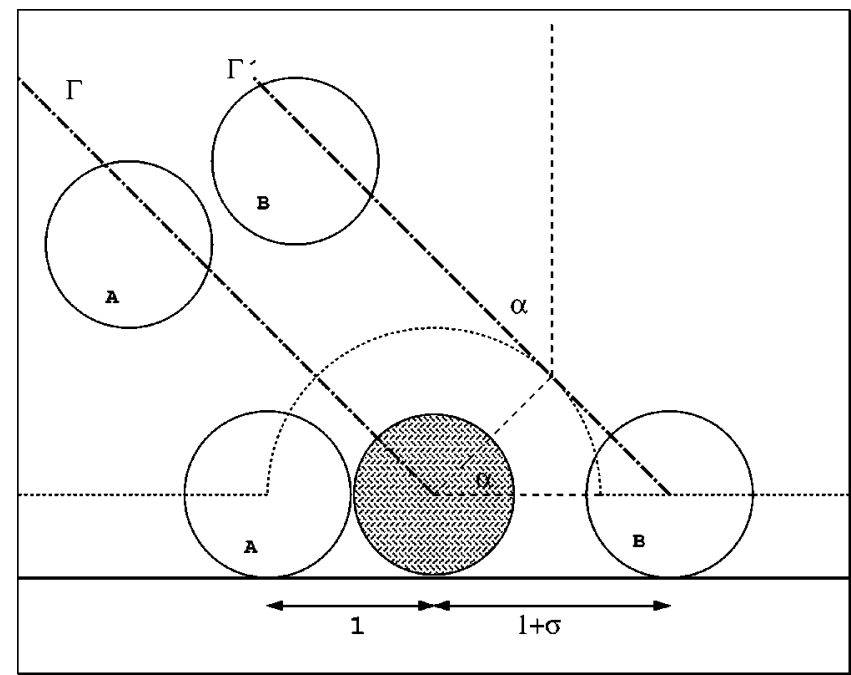

FIG. 3. Different possibilities of rolling for a disk arriving at the line in the presence of a preadsorbed particle. Particles with initial trajectory at the left of line $\Gamma$ roll to the left, whereas those with initial trajectory between the lines $\Gamma$ and $\Gamma^{\prime}$ roll to the right.

the adsorbed disk. At that point, it will continue its motion towards the substrate along a line parallel to its initial trajectory, which will be denoted $\Gamma^{\prime}$ (see Fig. 3). If no overlap is detected with a second disk, the new particle will be irreversibly adsorbed to the right of the fixed particle leaving an interval of length $\sigma$ between them. This mechanism is new respect to standard $\mathrm{BM}$, in which a particle rolling over a preadsorbed one cannot separate from it until it reaches the line.

Now, we will consider that a second disk is present on the line, at the right of disk 1, separated from it by a gap of length $l$ [see Fig. 4(a)]. If $l$ is larger than $1+\sigma$, then the trajectory of the incoming disk will follow the line $\Gamma^{\prime}$, being adsorbed at a distance $1+\sigma$ to the right of disk 1 without touching disk 2 (region I, Fig. 5). If the gap length $l$ is smaller, then the incoming particle will touch disk 2 before reaching the surface. However, it can either leave disk 1 along the separation line $\Gamma^{\prime}$ before touching disk 2, as shown in Figs. 4(a) and 4(b), or it can overlap disk 2 before arriving at that line [Figs. 4(c) and 4(d)].

Separation from disk 1 will not happen if the incident particle touches particle 2 before arriving at the separation line $\Gamma^{\prime}$. As shown in Figs. 4(c) and 4(d) this happens if the point of contact with the second particle is higher than the separation point, that is, if $\sqrt{1-(l+1)^{2} / 2}>\sin \alpha$. This gives the condition $0<l<2 \cos \alpha-1$, that can be fulfilled only for $\alpha<60^{\circ}(\sigma<1)$. Otherwise, the new particle will leave contact with particle 1 and follow a straight trajectory along the line $\Gamma^{\prime}$ before touching disk 2 . We describe separately both possibilities:

(a) $l>2 \cos \alpha-1$. The incoming particle leaves disk 1 before touching disk 2 , then it follows the line $\Gamma^{\prime}$ and it will touch particle 2 if $l<1+\sigma$. After this contact, the particle will roll over disk 2 to the left if the line $\Gamma^{\prime}$ is to the left of line $\Gamma_{2}$, that is, if $l>\sigma$ [see Fig. 4(a)]. Otherwise, it will roll to the right of disk 2 arriving to the gap situated at the right of the initial gap [Fig. 4(b)]; in this case, successive rolling over several particles at the right of disk 2 is possible. This situation occurs when the inequalities $2 \cos \alpha-1<l<\sigma$ are satisfied, and this can happen for $\alpha>45^{\circ}$ only.

(b) $0<l<2 \cos \alpha-1$. The incoming particle touches disk 2 before reaching the line $\Gamma^{\prime}$, then its motion depends on the relative location of the point of contact with respect to the separation line of particle $2, \Gamma_{2}$ [see Figs. 4(c) and 4(d)]. If the point of simultaneous contact with both disks is at the left of $\Gamma_{2}$ then the particle will remain trapped at that point. This happens, as can be seen in Fig. 4(c), if the height of that point is smaller than $\cos \alpha$, that is, if $l>2 \sin \alpha-1$. Nonlocal rolling to the right of particle 2 will appear if the opposite inequality is true, and this is possible for $\alpha>30^{\circ}$ only [see Fig. 4(d)].

In Fig. 5 we show a summary of the different rolling mechanisms for a disk rolling to the right of the leftmost preadsorbed particle delimiting a gap of length $l$ according to the previous discussion. From this figure, we can define two characteristic gap lengths for each value of the angle:

$$
l_{1}(\alpha)= \begin{cases}1, & \alpha \leqslant 60^{\circ} \\ \sigma, & \alpha \geqslant 60^{\circ}\end{cases}
$$

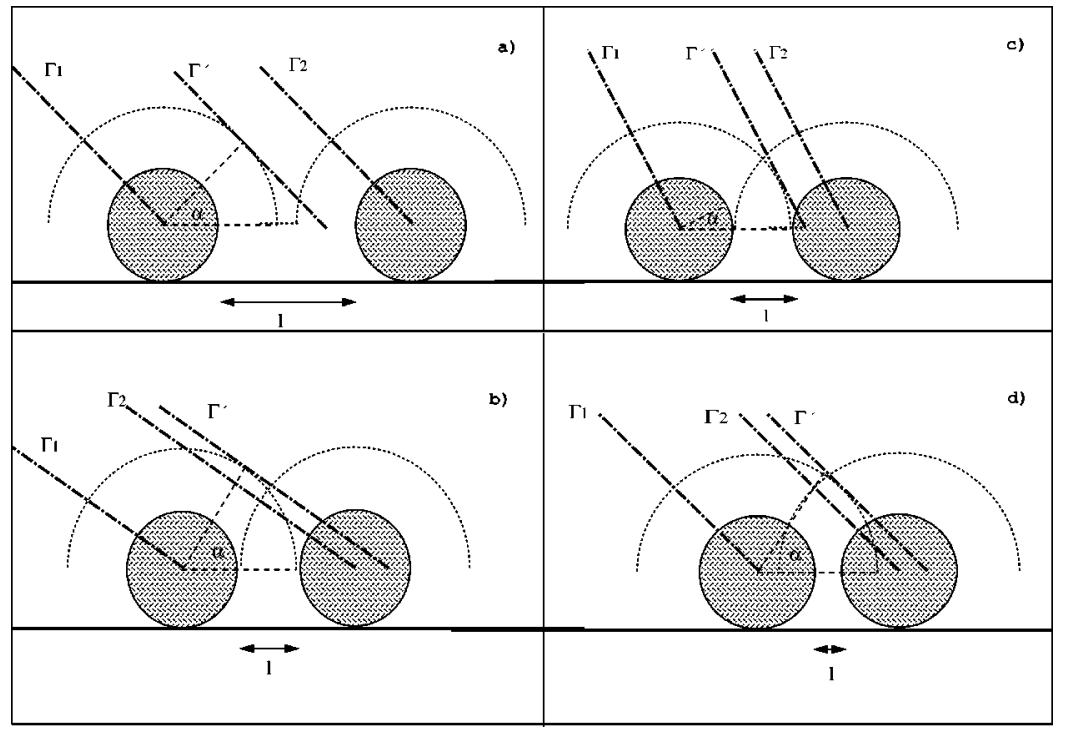

FIG. 4. Different possibilities of rolling for a disk arriving at the line in the presence of two pre-adsorbed particles. (a) and (b), $l>2 \cos \alpha$ -1 . A disk with initial trajectory between $\Gamma_{1}$ and $\Gamma^{\prime}$ will roll to the left and to the right of the second disk, respectively, after having left the contact with disk 1. (c) and (d), $l<2 \cos \alpha-1$. A disk with initial trajectory between $\Gamma_{1}$ and $\Gamma_{2}$ will be rejected or roll to the right of the second disk, respectively. 


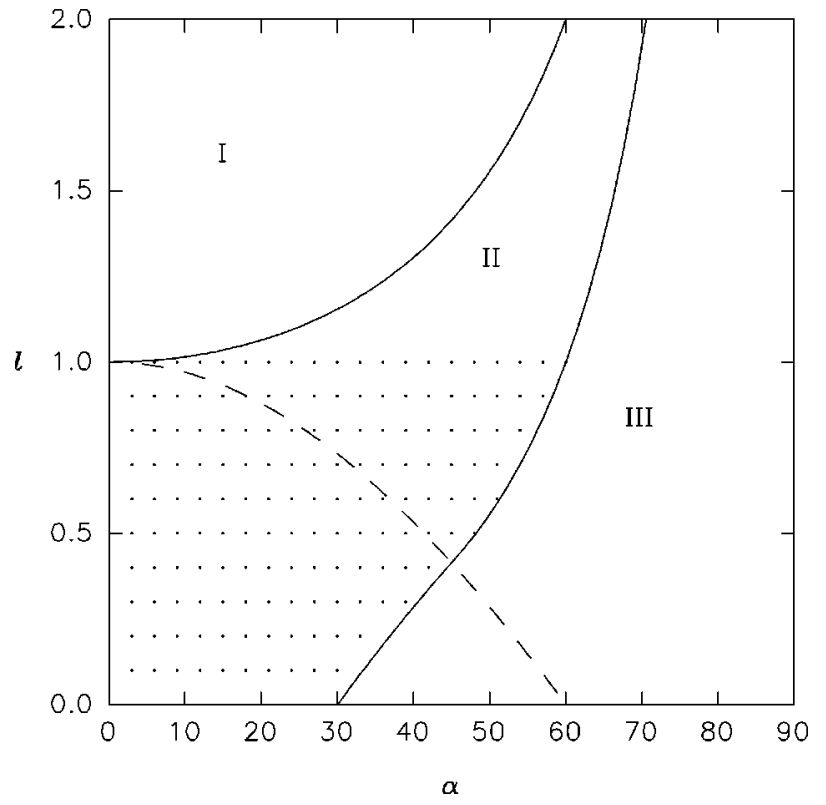

FIG. 5. Different rolling mechanisms, according to the previous figure, for a particle arriving along a direction forming an angle $\alpha$ with the normal, rolling to the right of disk 1 in the presence of a second disk on the line. The centers of disks 1 and 2 are separated by a distance $1+l$. In region I there is no contact with disk 2 , in region II the particle touches disk 2 and rolls to its left, and in region III the particle rolls to the right of disk 2, introducing nonlocal effects. The dashed line $l=2 \cos \alpha-1$ separates situations in which separation from disk 1 occurs before contact with disk 2 (region over that line) from those with successive rolling over both particles. The dotted area corresponds to situations where the incoming particle is rejected. Length is measured in diameter units, and $\alpha$ is expressed in degrees.

$$
l_{2}(\alpha)= \begin{cases}0, & \alpha \leqslant 30^{\circ}, \\ 2 \sin \alpha-1, & 30^{\circ} \leqslant \alpha \leqslant 45^{\circ}, \\ \sigma, & \alpha \geqslant 45^{\circ},\end{cases}
$$

We have shown that for arrival angles $\alpha>30^{\circ}$, an incoming disk the initial trajectory of which overlaps a gap of length $l<l_{2}(\alpha)$ will roll over the rightmost particle of the gap arriving at the next gap. This mechanism is not present in vertical BM and it implies that the adsorption rate at a given point may depend on the distribution of particles at arbitrary distance at its left. The adsorption kinetics becomes nonlocal in the sense that the final position of a particle on the line is not restricted to a position belonging to the initial gap in which the center of the incoming disk has been chosen. This means that the shielding property [1] that allows one to solve many one-dimensional adsorption problems is not satisfied if $\alpha>30^{\circ}$. In this regime there exists an interaction between gaps, and only for $\alpha<30^{\circ}$ it will be possible to obtain an analytic solution to this model.

Note that adsorption of new particles is possible only on gaps of length $l \geqslant l_{1}(\alpha)$, and therefore those particles arriving at gaps of length $l_{2}(\alpha) \leqslant l<l_{1}(\alpha)$ are rejected. For values of the angle $\alpha \geqslant 60^{\circ}$, one has $l_{1}=l_{2}$ and the rejection mechanism is suppressed: all the incoming particles will be able to reach the line while available intervals are present. This implies that the coverage will increase linearly until jamming, which will be reached in a finite time $t_{J}(\alpha)$, i.e., $\theta(t, \alpha)=t$ for $t \leqslant t_{J}(\alpha)$. Therefore, nonlocal adsorption deeply modifies the adsorption kinetics for large incident angles.

So far, we have not specified what happens to those particles that arrive exactly along the separating line $\Gamma$ of a preadsorbed disk. In standard BM this situation is irrelevant since only a fraction of zero measure of the incoming disks will follow these trajectories. Now the situation is different, because a finite fraction of the particles overlapping a disk will roll to its right trying to reach the line $\Gamma^{\prime}$. Once a particle has followed this path and it has been adsorbed forming a gap of length $\sigma$, the separation line of that particle, $\Gamma_{2}$, coincides with the line $\Gamma^{\prime}$ of the first particle. Any new particle rolling to the right over the first particle, and arriving at the line $\Gamma^{\prime}$, will follow the same path and will arrive along that separating line. As we have seen, this is possible only if the gap between the first particles, $l=\sigma$, is larger than $2 \cos \alpha-1$; i.e., for $\alpha>45^{\circ}$. Since an attractive interaction between the disks and the substrate will always exist, which makes it possible for the particles to be irreversibly adsorbed at the positions at which they arrive, we assume that such particles arriving along the separating line will roll to the left. Therefore, these particles will be rejected if $\sigma<1\left(45^{\circ}\right.$ $\left.<\alpha<60^{\circ}\right)$ and adsorbed if $\sigma>1\left(\alpha>60^{\circ}\right)$.

It is possible to find an analytic expression for certain physical quantities in this one-dimensional version of the ballistic model, using the same technique as in standard BM [6]. However, the method makes use of the shielding property of the kinetic one-dimensional models, and as we have discussed, this feature is absent due to the nonlocal adsorption mechanism for $\alpha>30^{\circ}$. Therefore, an exact analytic expression can be obtained only for $\alpha<30^{\circ}$. For $\alpha>30^{\circ}$ we will introduce a self-consistent description of the nonlocal mechanism that gives reasonably good results as we will see by comparison with computer simulations. We will present in Sec. III A the theoretical predictions, and we will compare them in Sec. III B with simulation results.

\section{A. Theoretical analysis}

Let us consider again the gap density $G(l, t)$. Due to the rolling mechanism [6], it contains now singular contributions. The evolution equations will couple the regular and singular components of the gap density. We introduce the decomposition of $G(l, t)$ into singular and regular parts as [19]

$$
\begin{aligned}
G(l, t)= & g(l, t)+g_{0}(t) \delta(l)+g_{\sigma}(t) \delta(l-\sigma) \\
& +g_{\sigma-1}(t) \delta(l-\sigma+1),
\end{aligned}
$$

where $g(l, t)$ refers to the regular contribution to the gap density, and $g_{0}(t), g_{\sigma}(t)$, and $g_{\sigma-1}(t)$ to the fraction of gaps of lengths $0, \sigma$, and $\sigma-1$, respectively, and where the latter appears for $\alpha>60^{\circ}$ only. Note that the singular contributions are related to the gap lengths through the rolling mechanisms.

We can now proceed to find the evolution equation for the gap densities. This can be done by looking at how gaps of length $l$ are destroyed and created from larger gaps, using the results of the preceding paragraphs. As a new ingredient with 
respect to the analysis of the standard models, we introduce a quantity $\Delta(t)$, which will account for the number of particles per unit time arriving to an interval from its left neighbor due to nonlocal adsorption. This mechanism introduces an "interaction', between the gaps, which otherwise would evolve independently. We assume that $\Delta(t)$ is the same for all the gaps, independently of their neighborhood. This means that we include the interaction between gaps in an effective or mean field way, and makes our analysis only approximate. A similar idea in the context of RSA with diffusion in a precursor layer is considered in Ref. [23], where the approach turns out to be exact.

The function $\Delta(t)$ will be determined from a selfconsistent condition obtained from the balance of particles arriving to the line. From the total number of particles arriving at the surface, a fraction $\phi$ will be adsorbed, while a fraction $R=1-\phi$ will be rejected. As we have seen, adsorption is only possible on gaps of length $l \geqslant l_{1}(\alpha)$; particles can arrive to one of such gaps through straight incident trajectories, at a total rate $l+1$, or after rolling from the left neighboring gap, at a rate $\Delta(t)$. Therefore, the rate at which particles arrive at each of these gaps is $l+1+\Delta(t)$, and the mean adsorption rate on the line is

$$
\phi(t)=\int_{l_{1}^{-}}^{\infty} d l[l+1+\Delta(t)] G(l, t) .
$$

On the other side, rejection of particles happens only in gaps of length $l_{2} \leqslant l<1$, where particles arrive at rate $l+1$ $+\Delta(t)$. The corresponding mean rejection rate is

$$
R(t)=\int_{l_{2}^{-}}^{l_{1}^{-}} d l[l+1+\Delta(t)] G(l, t) .
$$

Note that these integrals include the singular contributions to $G(l, t)$, and the minus sign in the integration limits indicate that the lower limit must be included in the integration interval, and the upper limit must be excluded. In particular, for $\alpha>60^{\circ}$ one has $l_{2}=l_{1}=\sigma$, and $R(t)=0$.

The self-consistent condition on $\Delta(t)$ can be obtained by imposing that the adsorption and rejection rates must add to one,

$$
\phi(t)+R(t)=\int_{l_{2}^{-}}^{\infty} d l[l+1+\Delta(t)] G(l, t)=1 .
$$

By combining this equation with the normalization condition Eq. (2.4), one obtains a more appealing form of the closure relation,

$$
\int_{0}^{l_{2}^{-}} d l(l+1) G(l, t)=\Delta(t) \int_{l_{2}^{-}}^{\infty} d l G(l, t) .
$$

The left member of this equation gives the total rate at which particles arrive at intervals of length $l<l_{2}(\alpha)$; all of these particles will roll over the rightmost particle limiting the gap, and constitute the source of the nonlocal effect. The right member of Eq. (3.6) gives the total rate at which the nonlocal effect drives particles towards gaps of length $l>l_{2}(\alpha)$, where they are finally adsorbed [if $l>l_{1}(\alpha)$ ] or rejected [if $l<l_{1}(\alpha)$ ]. For $\alpha<30^{\circ}, l_{2} \equiv 0$, which implies that also $\Delta(t)$
$=0$, since in this case nonlocal adsorption is not possible. In this region the calculations of the next subsection will provide an exact solution for the kinetics of the model.

The new singular adsorption mechanism for $\alpha>60^{\circ}$, represented by $g_{\sigma-1}$, induces a qualitatively different kinetics. Therefore, we will analyze separately the behavior of the inclined BM model for small and big angles.

\section{1. $\alpha<60^{\circ}$}

In order to derive the evolution equation for the gap density, we use the fact that direct adsorption without contact with adsorbed particles gives the same contributions that for the inclined RSA model, considered in Eqs. (2.1)-(2.3). Following the usual procedure [6], we introduce the contribution from rolling on preadsorbed particles. According to the different rolling mechanisms described in the preceding subsection, one finally arrives at the set of equations

$$
\begin{aligned}
\frac{\partial g_{1}(l, t)}{\partial t}= & -[l+1+\Delta(t)] g_{1}(l, t)+[1+\sigma+\Delta(t)] \\
& \times g_{1}(l+1+\sigma, t)+g_{1}(l+1, t)+\int_{l+1}^{\infty} d l^{\prime} g_{1}\left(l^{\prime}, t\right) \\
& +\int_{l+1+\sigma}^{\infty} d l^{\prime} g_{1}\left(l^{\prime}, t\right), \quad l \geqslant 1
\end{aligned}
$$

$$
\begin{array}{r}
\frac{\partial g_{2}(l, t)}{\partial t}=[1+\sigma+\Delta(t)] g_{1}(l+1+\sigma, t)+g_{1}(l+1, t) \\
+\int_{l+1}^{\infty} d l^{\prime} g_{1}\left(l^{\prime}, t\right)+\int_{l+1+\sigma}^{\infty} d l^{\prime} g_{1}\left(l^{\prime}, t\right), \\
\sigma<l<1 ;
\end{array}
$$

$$
\begin{aligned}
\frac{\partial g_{3}(l, t)}{\partial t}= & {[1+\sigma+\Delta(t)] g_{1}(l+1+\sigma, t)+[l+2+\Delta(t)] } \\
& \times g_{1}(l+1, t)+\int_{l+1+\sigma}^{\infty} d l^{\prime} g_{1}\left(l^{\prime}, t\right), \quad 0<l<\sigma ;
\end{aligned}
$$

$$
\begin{aligned}
\frac{d g_{0}(t)}{d t}= & \int_{1+\sigma}^{\infty} g_{1}\left(l^{\prime}, t\right) d l^{\prime} \\
& +\int_{1}^{1+\sigma}\left[l^{\prime}+1+\Delta(t)\right] g_{1}\left(l^{\prime}, t\right) d l^{\prime} \\
\frac{d g_{\sigma}(t)}{d t}= & {[1+\sigma+\Delta(t)] \int_{1+\sigma}^{\infty} g_{1}\left(l^{\prime}, t\right) d l^{\prime} }
\end{aligned}
$$

The first three equations give the evolution of the regular contribution to the gap density, and the last two the singular components. We have introduced the subscripts 1, 2, and 3 to distinguish the regular contribution, $g(l, t)$, in the three domains in which the kinetic mechanisms are different. Equation (3.7) takes into account that gaps of length $l>1$ may be destroyed by adsorption of a new disk arriving either di- 
rectly or after rolling over any of the limiting disks of the gap, and that they can be created from larger gaps either to the left or right of the incoming disk. Equation (3.8) considers that gaps smaller than 1 and larger than $\sigma$ cannot be destroyed, but are created through the same mechanisms as the larger gaps. Finally, Eq. (3.9) indicates that gaps smaller than $\sigma$ cannot be destroyed and cannot be created to the left of an incoming disk that adsorbs directly. The equations for the singular contributions are obtained taking into account that gaps of length 0 appear by rolling to the left of the right particle in gaps of length $l>1+\sigma$, and by adsorption of any particle arriving to a gap of length $l \in(1,1+\sigma)$, while intervals of length $\sigma$ are created by rolling of the incoming particle to the right of the leftmost disk of a gap of length $l$ $>1+\sigma$. In this region, if $\alpha<30^{\circ} \Delta \equiv 0$ since in this case moving to a gap different from the incident one is forbidden, and an exact analytic solution exists.

Equations (3.7)-(3.11) can be solved following the procedure we have introduced in the previous section. We should first solve the integrodifferential equation for $g_{1}(l, t)$, and then the other functions will be calculated from it by quadratures. We always start from an empty line, and therefore initially the density of gaps is zero. According to this initial condition, we introduce the ansatz

$$
g_{1}(l, t)=t^{2} \Omega(t) F(t) e^{-(l+1) t} .
$$

where $F(t)$ is the function obtained in the inclined RSA model, Eq. (2.7), and the dependence in $\sigma$ is implicit in both $F(t)$ and $\Omega(t)$. Introducing this expression for $g_{1}(l, t)$ in Eq. (3.31), and imposing that initially the substrate is empty, $\Omega(0)=1$, we find

$$
\begin{aligned}
\Omega(t, \sigma)= & \exp \left\{2-e^{-t}-e^{-(1+\sigma) t}\right. \\
& \left.-\int_{0}^{t} d \tau \Delta(\tau)\left[1-e^{-(1+\sigma) \tau}\right]\right\} .
\end{aligned}
$$

Now, we can also determine $g_{2}(l, t)$ and $g_{3}(l, t)$, as well as the singular contributions, as quadratures, by substituting Eq. (3.12) in Eqs. (3.8)-(3.11). One arrives at

$$
\begin{aligned}
g_{2}(l, t)= & \int_{0}^{t} \tau \Omega(\tau) F(\tau) e^{-(l+2) \tau} \\
& \times\left\{\tau+1+[\tau(1+\sigma+\Delta(\tau))+1] e^{-\sigma \tau}\right\} d \tau,
\end{aligned}
$$

$$
\begin{aligned}
g_{3}(l, t)= & \int_{0}^{t} \tau \Omega(\tau) F(\tau) e^{-(l+2) \tau}\{[l+2+\Delta(\tau)] \tau \\
& \left.+e^{-\sigma \tau}[1+(1+\sigma+\Delta(\tau)) \tau]\right\} d \tau, \\
g_{0}(t)= & \int_{0}^{t} \Omega(\tau) F(\tau) e^{-2 \tau}\left\{1+[2+\Delta(\tau)] \tau-e^{-\sigma \tau}\right. \\
& \times[1+(1+\sigma+\Delta(\tau)) \tau]\} d \tau,
\end{aligned}
$$

$$
g_{\sigma}(t)=\int_{0}^{t} \tau \Omega(\tau) F(\tau) e^{-(\sigma+2)}[1+\sigma+\Delta(\tau)] d \tau .
$$

Letting time go to infinity, a jamming state is reached in which there are no gaps of length larger than 1 . Note that in the limit $\sigma \rightarrow 0$, the singular contributions $g_{0}$ and $g_{\sigma}$ are identical, and the sum of both gives the delta function characteristic of BM. On the other hand, the function $g_{3}$ disappears when performing this limit. Therefore, the behavior of $G(l, t)$ at contact $(l \rightarrow 0)$ will depend on $\sigma$.

We can now derive an expression for the time evolution of the coverage. Since the gap density $G(l, t)$ defined in Eq. (3.2) includes all the interparticle gaps, including the singular contributions, its integral respect to $l$ gives the total number density of gaps and therefore the number density of particles [19]. Integrating it, we arrive at

$$
\theta(t)=\int_{0}^{\infty} d l G(l, t)=\int_{0}^{t} \Omega(\tau) F(\tau) e^{-2 \tau}[1+(2+\Delta(\tau)) \tau] d \tau
$$

which has the same form as the one obtained in standard BM [6], with the addition of the $\Delta(\tau)$ term. The dependence on $\sigma$ is now implicit in $\Omega(t), F(t)$, and $\Delta(t)$.

To obtain $\Delta(t)$, one has to solve simultaneously the selfconsistent condition expressed by Eq. (3.5) or (3.6) and the integrals (3.14)-(3.17) for the different contributions to $G(l, t)$. For $\alpha<30^{\circ}$ one has $l_{2}=0$, and Eq. (3.6) implies that $\Delta(t)=0$, which is consistent with the absence of nonlocal adsorption at these incident angles. For $\alpha>30^{\circ}, \Delta(t)$ must be obtained numerically using the self-consistent condition Eq. (3.5).

At short times, one can expand all quantities in positive powers of time, and the first coefficients can be explicitly obtained. One has, for example, $\Delta(t)=\Delta_{1} t+O\left(t^{2}\right)$, with $\Delta_{1}=\left[1+l_{2}\left(1+l_{2} / 2\right)\right] / 2$ if $30^{\circ}<\alpha<60^{\circ}$, and $\Delta_{1}=0$ if $\alpha$ $<30^{\circ}$. For the coverage one then obtains

$$
\theta(t)=t-\left(\frac{5}{2}+\frac{\sigma}{2}+\frac{\sigma^{2}}{4}-\Delta_{1}\right) \frac{t^{3}}{3}+O\left(t^{4}\right) .
$$

Note that the effects of the inclination, including the nonlocal rolling for $\alpha>30^{\circ}$, appear in the third order in time, since three particles are needed to make clear the rolling mechanisms; the second-order term is missing because if only one particle is adsorbed on the substrate, all the incident particles can reach the line.

At large times, all quantities approach an asymptotic (jamming) value exponentially fast, as in standard BM. For example, for the coverage one has

$$
\theta_{J}-\theta(t) \approx K \frac{e^{-\left(2+\Delta_{J}\right) t}}{t},
$$

where both the amplitude $K$ and the asymptotic value of $\Delta(t), \Delta_{J}$, depend on the incident angle $\alpha$.

The maximum fraction of the line covered with particles can be expressed as a quadrature, extending the integration 
interval in Eq. (3.18) to infinity. For small values of the inclination angle $\alpha$, this quantity can be expanded as a power series of $\sigma$, giving

$$
\begin{aligned}
\theta^{\infty}(\sigma)= & \int_{0}^{\infty} d t \exp \left\{-2 t+2-2 e^{-t}-2 \int_{0}^{t} \frac{1-e^{-u}}{u} d u\right\} \\
& \times(1+2 t)\left\{1+\left[e^{-t}(1+t)-1\right] \sigma+\left[e^{-2 t}(1+t)^{2}\right.\right. \\
& \left.\left.-e^{-t}\left(t^{2}+3 t+3\right)+2\right] \sigma^{2} / 2\right\}+O\left(\sigma^{3}\right) \\
= & 0.808653-0.110518 \sigma-0.000424898 \sigma^{2}+O\left(\sigma^{3}\right),
\end{aligned}
$$

where in the last equation we have given numerical expressions for the exact result to give an idea of the order of magnitude of the corrections introduced by $\sigma$. Note that for $\alpha \leqslant 30^{\circ}$ (small $\left.\sigma\right), \Delta(t)=0$, and the previous analysis is exact. As we will show in Sec. III B, the results obtained from the self-consistent analysis constitute also a good approximation for angles $\alpha \in\left[30^{\circ}, 60^{\circ}\right]$.

\section{2. $\alpha>60^{\circ}$}

For $\alpha>60^{\circ},(\sigma>1)$, the nonlocal mechanism becomes essential. One has now $l_{1}=l_{2}=\sigma$ and, as shown in Eq. (3.4), the rejection rate vanishes. The self-consistency condition (3.5) reduces now to $\phi=1$, showing that the kinetic law is trivial; the only kinetic quantity to be determined is the finite time $t_{J}$ at which the jamming is reached, which is numerically equal to the jamming coverage $\theta_{J}$.

The rate equations for the gap density show also slight modifications respect to those corresponding to the case $\alpha<60^{\circ}$, Eqs. (3.7) $-(3.11)$. First, the decomposition (3.2) must include now the singular contribution $g_{\sigma-1}(t)$, corresponding to gaps of length $\sigma-1$ that are created by particles adsorbed in gaps of length $\sigma$. Now, only intervals of length $l \geqslant \sigma$ can accept new particles, because particles arriving at smaller intervals will roll over the rightmost particle, going to the next interval. All particles arriving at intervals of length $l \in[\sigma, \sigma+1]$ will roll over the limiting particles, being adsorbed at contact with the rightmost one. As a consequence, Eq. (3.7) is valid for $l>l_{1}=\sigma$, whereas Eqs. (3.8)(3.11) now read

$$
\begin{aligned}
& \frac{\partial g_{2}(l, t)}{\partial t}=[1+\sigma+\Delta(t)] g_{1}(l+1+\sigma, t)+[l+2+\Delta(t)] \\
& \times g_{1}(l+1, t)+\int_{l+1+\sigma}^{\infty} d l^{\prime} g_{1}\left(l^{\prime}, t\right), \\
& \sigma-1<l<\sigma \\
& \frac{\partial g_{3}(l, t)}{\partial t}=[1+\sigma+\Delta(t)] g_{1}(l+1+\sigma, t) \\
& +\int_{l+1+\sigma}^{\infty} d l^{\prime} g_{1}\left(l^{\prime}, t\right), \quad l<\sigma-1
\end{aligned}
$$

$$
\begin{gathered}
\frac{d g_{0}(t)}{d t}=\int_{1+\sigma}^{\infty} g_{1}\left(l^{\prime}, t\right) d l^{\prime} \\
+\int_{\sigma}^{1+\sigma}\left[l^{\prime}+1+\Delta(t)\right] g_{1}\left(l^{\prime}, t\right) d l^{\prime} \\
+[1+\sigma+\Delta(t)] g_{\sigma}(t) ; \\
\frac{d g_{\sigma}(t)}{d t}=[1+\sigma+\Delta(t)]\left\{\int_{1+\sigma}^{\infty} g_{1}\left(l^{\prime}, t\right) d l^{\prime}-g_{\sigma}(t)\right\} \\
\frac{d g_{\sigma-1}(t)}{d t}=[1+\sigma+\Delta(t)] g_{\sigma}(t) .
\end{gathered}
$$

These equations can be solved following the same procedure used for $\alpha<60^{\circ}$. Now, Eq. (3.12) is still valid for $l>\sigma$, whereas for the other components we have

$$
\begin{aligned}
g_{2}(l, t)= & \int_{0}^{t} \tau \Omega(\tau) F(\tau) e^{-(l+2) \tau}\{[l+2+\Delta(\tau)] \tau \\
& \left.+[1+\tau(1+\sigma+\Delta(\tau))] e^{-\sigma \tau}\right\} d \tau
\end{aligned}
$$

$g_{3}(l, t)=\int_{0}^{t} \tau \Omega(\tau) F(\tau) e^{-(l+2+\sigma) \tau}\{[1+\sigma+\Delta(\tau)] \tau+1\} d \tau$

$$
\begin{aligned}
g_{0}(t)= & \int_{0}^{t} \Omega(\tau) F(\tau) e^{-(2+\sigma) \tau}\left\{e^{\tau}-1+\tau[1+\sigma+\Delta(\tau)]\right. \\
& \left.\times\left[e^{\tau}-e^{-\int_{\tau}^{t}[1+\sigma+\Delta(u)] d u}\right]\right\} d \tau \\
g_{\sigma}(t)= & e^{-(2+\sigma) t} \int_{0}^{t} \tau \Omega(\tau) F(\tau) \\
& \times[1+\sigma+\Delta(\tau)] e^{\int_{\tau}^{t}[1-\Delta(u)] d u} d \tau \\
g_{\sigma-1}(t)= & \int_{0}^{t} \tau \Omega(\tau) F(\tau)[1+\sigma+\Delta(\tau)] e^{-(2+\sigma) \tau} \\
& \times\left\{1-e^{-\int_{\tau}^{t}[1+\sigma+\Delta(u)] d u}\right\} d \tau
\end{aligned}
$$

Introducing these expressions in Eq. (3.6), the selfconsistent condition can be written as

$$
\begin{aligned}
\phi(t)= & {[1+\sigma+\Delta(t)] g_{\sigma}(t)+\Omega(t) F(t) e^{-(1+\sigma) t} } \\
& \times\{1+[1+\sigma+\Delta(t)] t\}=1
\end{aligned}
$$

Note that the nonlocal term $\Delta(t)$ is necessary to satisfy this condition. Neglecting this effect would lead to an unrealistic kinetics. As a consequence, the approach to the jamming limit is very different from the situation when $\alpha<60^{\circ}$. Now, when $t \rightarrow t_{J}, G(l, t) \rightarrow 0$ for $l \geqslant l_{2}=\sigma$ and, according to Eq. (3.6), $\Delta(t)$ diverges. From Eq. (3.32) one can obtain the divergent behavior,

$$
\Delta(t) \approx \frac{\left[1-e^{-(1+\sigma) t_{J}}\right]^{-1}}{t_{J}-t}, \quad t \rightarrow t_{J}
$$




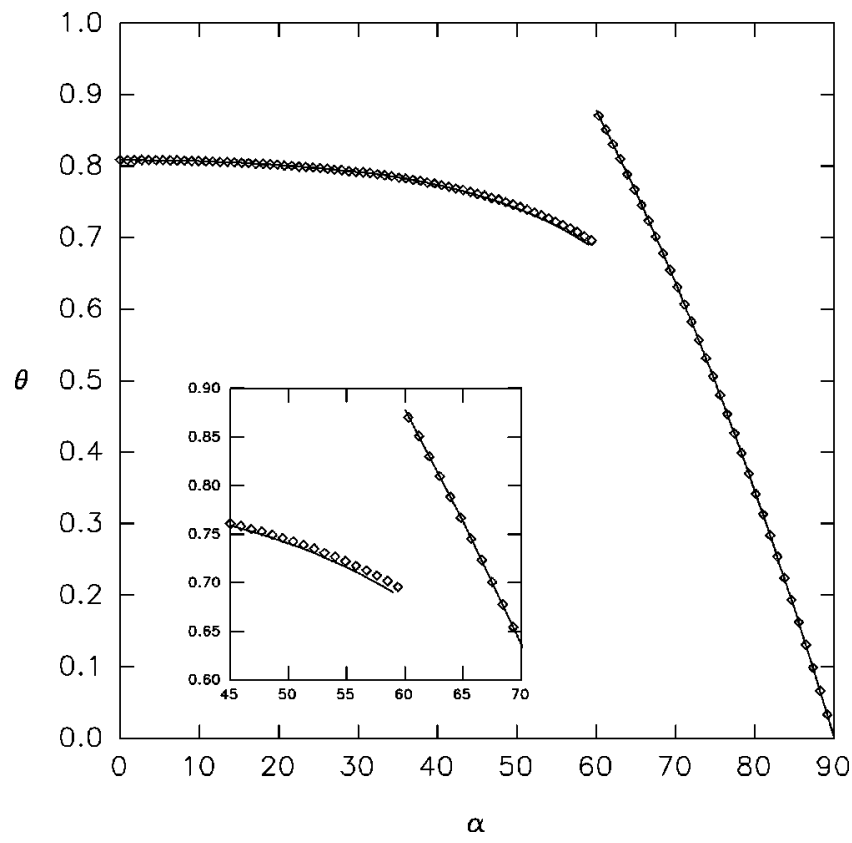

FIG. 6. Jamming limit of the inclined BM as a function of the incident angle $\alpha$, expressed in degrees. The line corresponds to the analytic solution, which is exact for $\alpha<30^{\circ}$ and approximate for $\alpha>30^{\circ}$, and the symbols correspond to the simulation results, in which all possible processes affecting the arrival of spheres at the substrate have been taken into account. In the inset, detail of the region where the discontinuity takes place.

This divergence reflects the fact that, at jamming, the incoming particles remain for ever rolling over the saturated substrate.

In the limit $\alpha \rightarrow 90^{\circ}$, the direction of the incident particles is near parallel to the surface, and one has $\sigma \rightarrow \infty$. In this situation one has two disparate length scales, the radius of the particles and the length of their "shadows," $\sigma$; correspondingly, there exist two time scales: the characteristic time scale for arrival of a new particle per unit length ( 1 in non-dimensional units), and the characteristic time of jamming (of order $\sigma^{-1}$ ). The first time scale is irrelevant in the limit $\sigma \rightarrow \infty$, and all the functions of time approach a scaling form of the type $f(t) \approx \sigma^{a} \widetilde{f}(\sigma t)$. Therefore one has

$$
\begin{gathered}
\Delta(t) \approx \sigma \widetilde{\Delta}(\sigma t), \\
\Omega(t) \approx \widetilde{\Omega}(\sigma t), \\
g_{\sigma}(t) \approx \sigma^{-1} \widetilde{g}_{\sigma}(\sigma t) .
\end{gathered}
$$

According to these scalings, the coverage scales as $\sigma^{-1}$. By numeric integration one can obtain the asymptotic approximation for the jamming coverage

$$
\theta^{\infty}(\sigma) \approx 2.1238 \sigma^{-1}+O\left(\sigma^{-2}\right) .
$$

\section{B. Simulation results}

We have performed numerical simulations of the sequential adsorption of disks of diameter one on a line of length $L=10^{3}$, using the rules of the inclined BM described at the beginning of this section: the initial position of each disk is

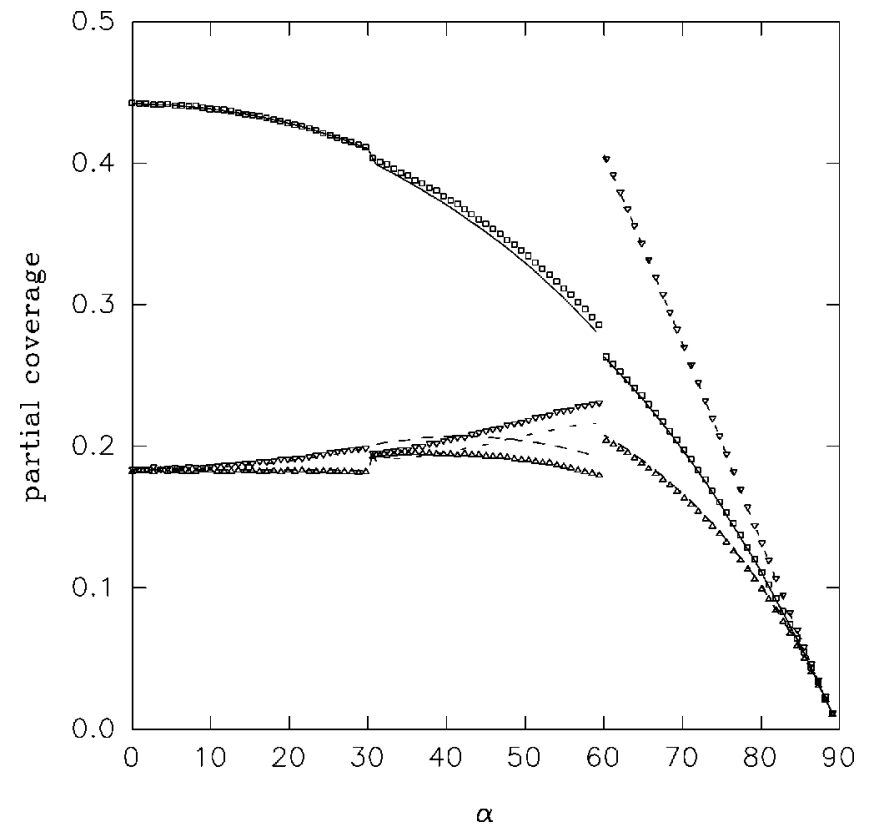

FIG. 7. Contribution to the jamming coverage of particles that have arrived at the surface by direct adsorption (squares), and of those the last movement of which has been a roll to the right (triangles) and to the left (inverted triangles), as a function of the incident angle, expressed in degrees. The lines show the results obtained from the self-consistent approach.

chosen randomly on a line at height 1 over the adsorbing line, and its inclined trajectory is generated from that point, rolling over as many disks as they are allowed before either reaching the substrate or being rejected. We start with an empty line, which is sequentially filled until jamming is achieved. In Fig. 6 we show the jamming coverage as a function of the angle of arrival of disks obtained after averaging over $10^{3}$ simulations for each value of the angle. The simulation results are compared with the results of the selfconsistent calculations.

As a major feature one can see that, despite the approximate character of the analytic solution for $\alpha>30^{\circ}$, it reproduces the jamming coverage quite well, except in the neighborhood of $\alpha=60^{\circ}(\sigma=1)$. When increasing the incident angle $\alpha$ from 0 , initially one observes a decrease of the jamming, indicating that a finite fraction of particles roll to the right of preadsorbed spheres, creating gaps of increasing length $\sigma$ that cannot be covered by additional particles. For angles greater than $30^{\circ}$, particles can roll over preadsorbed spheres, increasing the probability that a gap of length $\sigma$ is produced. This effect is not completely taken into account by the self-consistent equation, which slightly underestimates the jamming coverage, specially for angles near $60^{\circ}$. Close to $\sigma=1^{-}$, the jamming has decreased $15 \%$ with respect to standard BM. At $\sigma=1^{+}$, a jump in the jamming is observed, which is well reproduced by the analytic model, meaning that the increase is basically due to the possibility of the adsorption of incoming particles on gaps of length $\sigma$. In fact, the magnitude of the jump approximately equals the number of such intervals at jamming for $\sigma=1^{-}, g_{\sigma}(\infty)$.

For angles larger than $60^{\circ}$ a rapid decrease of the coverage is observed, which is well reproduced by the selfconsistent solution. In this region rolling to the right of an 


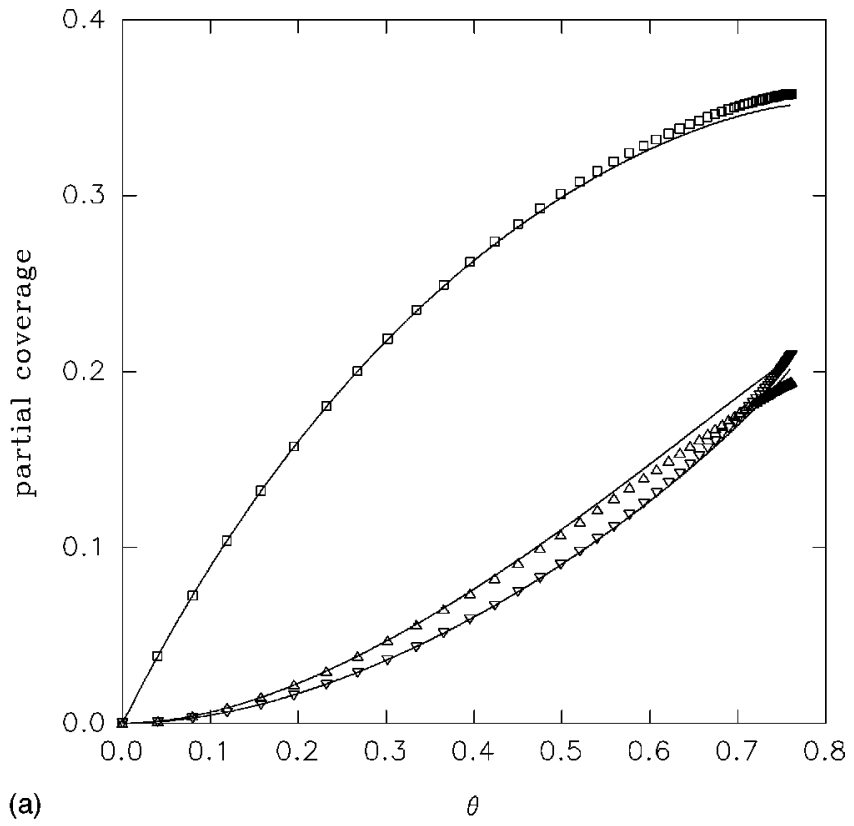

(a)

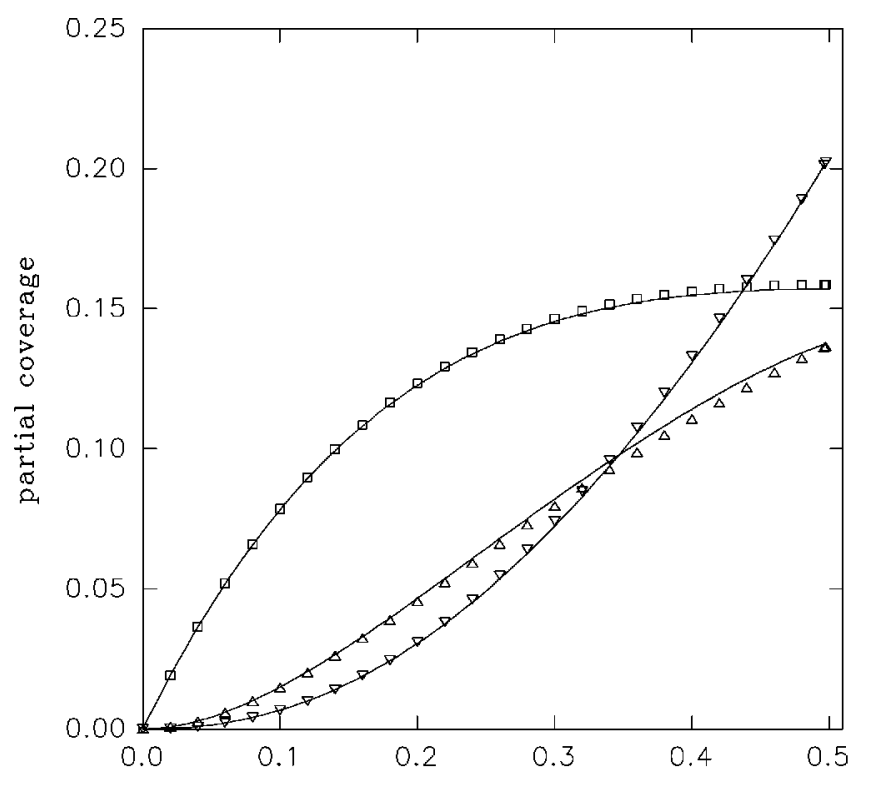

(b)

$\theta$

FIG. 8. Evolution of the different contributions to the coverage, $\theta_{D}$ (squares), $\theta_{R}$ (triangles), and $\theta_{L}$ (inverted triangles) as a function of the total coverage for (a) $\alpha=45^{\circ}$ and (b) $\alpha=75^{\circ}$. The lines show the self-consistent results.

adsorbed disk is the most favorable event, enhancing the formation of larger gaps. For $\alpha \rightarrow 90^{\circ}$, the coverage vanishes in the way predicted by the asymptotic limit of the selfconsistent theory, Eq. (3.37).

In order to gain more insight on the importance of the different mechanisms, we have also observed the average last movement of the incoming disks. In Fig. 7 we show the contribution to the jamming coverage due to particles that have arrived to the line directly, $\theta_{D}$, those that have arrived after having rolled the last time to the right, $\theta_{R}$, and those whose last movement has been to roll to the left of a preadsorbed particle, $\theta_{L}$. They are related to the singular components of the gap distribution function, $\theta_{L}=g_{0}$, and $\theta_{R}=g_{\sigma}$ $+g_{\sigma-1}$.

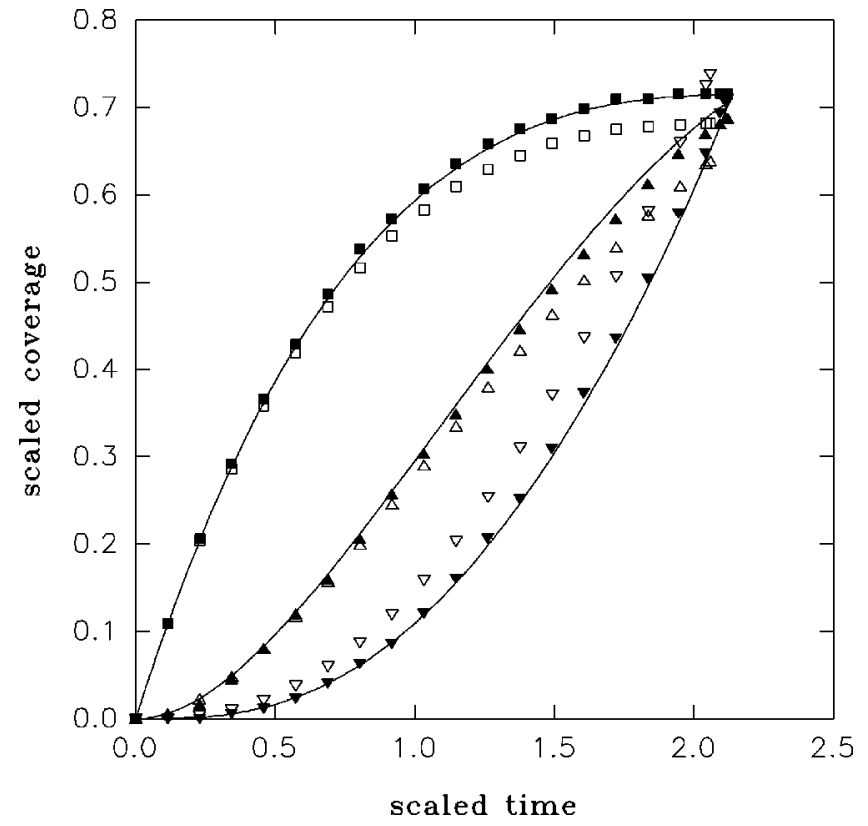

FIG. 9. Scaling plot of the different contributions, $\sigma \theta_{D}$ (squares), $\sigma \theta_{R}$ (triangles), and $\sigma \theta_{L}$ (inverted triangles) as function of the scaling time $\sigma t=\sigma t$ for $\alpha=85^{\circ}$ (hollow symbols) and $\alpha$ $=89^{\circ}$ (filled symbols). The lines show the scaling curves obtained from the self-consistent approach.

For $\alpha=0^{\circ}$ the system is symmetric and $\theta_{L}=\theta_{R}$; for $\alpha$ $<30^{\circ}, \theta_{L}$ increases slightly due to the fact that, although rolling to the right is more probable than rolling to the left, particles rolling to the right have a larger probability of being rejected because they need a larger length to be adsorbed.

At $\alpha=30^{\circ}$ the nonlocal mechanism starts to appear, favoring rolling to the right, and according to the simulation results, one recovers the equality $\theta_{L} \approx \theta_{R}$. Since more particles are now able to reach the line after rolling, the fraction which do it through direct adsorption decreases. Despite rolling to the right is now favored, the fraction of disks that adsorb after rolling to the left increases with the angle for the same reason as before. The self-consistent solution correctly reflects this tendency, but does not reproduce accurately the value of the partial contributions $\theta_{L}$ and $\theta_{R}$.

At $\alpha=60^{\circ}$, particles arriving to gaps of length $\sigma$ are accepted, producing a large increase in $\theta_{L}$. This discontinuity is directly related to the number of gaps of length $\sigma$ present on the substrate for $\alpha=60^{\circ}$, which could not be filled for $\alpha<60^{\circ}$. Now, the destruction of these gaps gives raise to new gaps of length $\sigma-1$ and 0 . These lasts in turn induce the rolling to the right of subsequent adsorbing particles, increasing also $\theta_{R}$. This also implies that the number of particles which adsorb directly decreases. For these angles, the self-consistent solution seems to be near exact.

The self-consistent solution reproduces well also the time evolution of the different components of the coverage, as shown in Fig. 8. At short times, direct adsorption is always dominant, as expected, and rolling to the right is more probable than rolling to the left. However, at longer times, rolling becomes dominant, and rolling to the left becomes more important than rolling to the right.

Finally, in Fig. 9 we show how the simulation results for the different contributions to the coverage approach the scal- 
ing functions obtained from the self-consistent solutions (3.29)-(3.31). Note that in this limit, the contributions $\theta_{L}$ and $\theta_{R}$ seem to be near equal at jamming, as predicts the self-consistent solution.

\section{CONCLUSION}

In this paper we have studied the kinetic properties of the shadow models, which describe the adsorption of particles when an additional field favors the motion of the adsorbing particles in a direction parallel to the substrate. Although it is known that diffusion [20], the effect of hydrodynamic interactions [21] or coadsorption [22] may become relevant, we have focused on the modifications that such a field introduces on the excluded volume interactions between the adsorbing and preadsorbed particles, and have therefore neglected a detailed description of the transport process from the bulk liquid. From this point of view, the external field can model either a shear flow, an external electric field parallel to the substrate or the adsorption process in presence of gravity on an inclined substrate. In all cases, adsorbing particles arrive at the surface along trajectories that form an angle $\alpha$ with the normal to the substrate. We have shown the excluded volume interactions are sensitive to the applied field, and we have analyzed their implications on the adsorption kinetics.

We have first focused on a model that evolves according to rules analogous to those of the RSA model, which is considered to represent the kinetics for diffusing colloidal particles. We have seen that both the kinetics and the jamming coverage are sensitive to the external field. In particular, the jamming decreases considerably when increasing $\alpha$. We have also seen that the nonequilibrium effects characteristic of these irreversible models start at lower coverage with respect to standard RSA due to the asymmetry induced by the external field.

We have also considered a kinetic model that evolves according to $\mathrm{BM}$ rules, which is adequate in the case of a strong field. In this case, the external field induces new processes. In particular, adsorption may become nonlocal, in the sense that an adsorbing particle may roll over a number of preadsorbed particles before either being adsorbed or rejected. This process induces an effective interaction between gaps, and therefore an exact analytic solution is restricted to incident angles $\alpha<30^{\circ}$. For higher angles, we have introduced a self-consistent model that accounts partially for the nonlocal mechanisms. We have compared the results predicted by this model with the results obtained from numerical simulations, showing that the differences are not too large. The self-consistent approach introduces only quantitative corrections for $\alpha<60^{\circ}$, but it is essential to describe the qualitative changes in the kinetics for $\alpha>60^{\circ}$.

\section{ACKNOWLEDGMENTS}

I.P. acknowledges the FOM for financial support, and the FOM Institute for its hospitality. J.B. and J.M.R. have been supported by the DGICYT of the Spanish Government under Grant Nos. PB94-0718 and PB95-0881, respectively.
[1] J. Evans, Rev. Mod. Phys. 65, 1281 (1993).

[2] J. Feder and I. Giaever, J. Colloid Interface Sci. 78, 144 (1980).

[3] I. Pagonabarraga, P. Wojtaszczyk, M. Rubí, B. Senger, P. Schaaf, and J.-C. Voegel, J. Chem. Phys. 105, 7815 (1996).

[4] G. Tarjus, P. Schaaf, and J. Talbot, J. Stat. Phys. 63, 167 (1991).

[5] E. L. Hinrichsen, J. Feder, and T. Jøssang, J. Stat. Phys. 44, 793 (1986)

[6] J. Talbot and S. Ricci, Phys. Rev. Lett. 68, 958 (1992); R. Jullien and P. Meakin, J. Phys. A 25, 1891 (1992).

[7] J. J. Ramsden, Phys. Rev. Lett. 71, 295 (1993); J. Stat. Phys. 73, 853 (1993).

[8] J. M. Meinders and H. J. Busscher, Colloid Polymer Sci. 272, 478 (1994).

[9] C. A. Monning and R. T. Kennedy, Anal. Chem. 66, 280R (1994).

[10] R. L. St.Claire, Anal. Chem. 68, 569R (1996).

[11] I. Pagonabarraga, J. Bafaluy, and J. M. Rubí, Phys. Rev. Lett. 75, 461 (1995)

[12] D. Boyer, J. Talbot, G. Tarjus, P. R. Van Tassel, and P. Viot,
Phys. Rev. E 49, 5525 (1994).

[13] D. H. Linares, R. H. López, and V. D. Pereyra, J. Phys. A 31, 1165 (1998).

[14] Handbook of Mathematical Functions, edited by M. Abramowitz and I. A. Stegun (Dover, New York, 1972).

[15] Y. Pomeau, J. Phys. A 13, L193 (1980).

[16] C. M. Bender and S. A. Orszag, Advanced Mathematical Methods for Scientists and Engineers (McGraw-Hill, Singapore, 1978).

[17] B. Widom, J. Chem. Phys. 44, 3888 (1966).

[18] P. Schaaf and J. Talbot, Phys. Rev. Lett. 62, 175 (1989).

[19] P. Viot, G. Tarjus, and J. Talbot, Phys. Rev. E 48, 480 (1994).

[20] F. J. Bafaluy, B. Senger, J.-C. Voegel, and P. Schaaf, Phys. Rev. Lett. 70, 623 (1993).

[21] I. Pagonabarraga and J. M. Rubí, Phys. Rev. Lett. 73, 114 (1994).

[22] M. R. Oberholzer, N. J. Wagner, and A. Lenhoff, J. Chem. Phys. 107, 9157 (1997); J. Faraudo and J. Bafaluy (unpublished).

[23] G. J. Rodgers and J. A. N. Filipe, J. Phys. A 30, 3449 (1997). 